\title{
Expression Level of FUT1 Gene in Different Pig Populations and its Relationship with ETEC F18 Resistance
}

\author{
Liu $\mathrm{Y}^{1}$, Xia RW ${ }^{1}$, Yin XM ${ }^{1}$, Huo YJ ${ }^{1}, \mathrm{Zhu} \mathrm{GQ}^{2}$, Wu SL ${ }^{1}$, Bao WB ${ }^{1 *}$ \\ ${ }^{1}$ Key Laboratory for Animal Genetics, Breeding, Reproduction and Molecular Design of Jiangsu Province, College of Animal Science and Technology, Yangzhou \\ University, Yangzhou Jiangsu, 225009 China \\ ${ }^{2}$ College of Veterinary Medicine, Yangzhou University, Yangzhou Jiangsu, 225009 China
}

Received: June 11, 2015; Accepted: August 21, 2015; Published: September 16, 2015

*Corresponding author: Wen Bin Bao, Key Laboratory for Animal Genetics, Breeding, Reproduction and Molecular Design of Jiangsu Province, College of Animal Science and Technology, Yangzhou University, Yangzhou Jiangsu, 225009 China, E-mail: wenbinbao74@yahoo.com

\begin{abstract}
F18-fimbriated Escherichia coli are associated with porcine post weaning diarrhea and edema disease. Some pigs show inherent resistance to F18 ETEC infection and this is associated with a G/A mutation at position M307 of the alpha $(1,2)$ - fucosyltransferase (FUT1) gene. Pigs with genotype AA are resistant to ETEC F18 and pigs with genotype GG or AG are susceptible to ETEC F18 infection. So the M307 of FUT1 gene has been proposed as a genetic marker to distinguish the E. coli F18 resistant from susceptible phenotypes in some imported pigs. The objective of this study was to investigate the different expression levels of FUT1 among Yorkshire, Meishan, and E. coli F18-resistant Sutai groups, especially in Meishan. The results showed that FUT1 was expressed consistently in 11 tissues in the three populations, with relatively high level in the lungs, stomach and gastrointestinal tract. The expression was highest in E. coli F18resistant Sutai pigs, followed by Yorkshire and Meishan. Considering that the biological processes and pathways FUT1 gene involved in was related to glycosphingolipid biosynthesis, we can speculate that higher expression of FUT1 in jejunum and duodenum is beneficial to the formation of receptors to $E$. coli F18. Besides, Meishan piglets may have its characteristic immune system for the infection of $E$. coli F18.
\end{abstract}

Keywords: Escherichia coli F18; FUT1; Gene expression; Weaning piglets

\section{Introduction}

F18- fimbriated Escherichia coli are associated with porcine post-weaning diarrhea. The presence of $E$. coli F18 receptor on small intestinal villi is the essential requirement for the adhesion and colonization. At present, there are a lot of researches on the receptor of E. coli F18, and the genetic locus for this receptor has been mapped to porcine chromosome 6 (SSC6), based on its close linkage to the S locus and other loci of the halothane (HAL) linkage group [1]. Coddens, et al. [2] found that F18-fimbriated E. coli selectively interact with glycosphingolipids having blood group $\mathrm{ABH}$ determinants on type 1 core, and blood group $\mathrm{A}$ type 4 heptaglycosylceramide. Alpha(1,2)-fucosyltransferases (FUTs) are key enzymes involved in the formation of blood group antigens of the porcine AO blood group system, which corresponds to the human $\mathrm{ABO}$ blood group system [3]. A pig $\alpha(1,2)$-fucosyltransferase exhibiting $\alpha(1,2)$-fucosylation of glycolipid and glycoprotein acceptors has been purified from submaxillary gland mucin [4]. Thurin and Blaszczyk-Thurin $M$ [5] identified this enzyme as the homologue of the human Secretor enzyme. Recent studies indicated that FUT1 gene was important in the synthesis of the structure that was beneficial to the adhesion between E. coli F18 fimbriated bacteria and the small intestinal wall [6].

By linkage analysis, Meijerink, et al.[7] estimated that the FUT1 gene polymorphism was less than 1 centimorgan from the $S$ and E. coli F18 receptor loci. Therefore, FUT1 gene was regarded as a good candidate for gene controlling the expression of the receptor for E. coli F18 bacteria. Sequencing of the FUT1 gene revealed a polymorphism ( $\mathrm{G}$ or $\mathrm{A}$ ) at nucleotide 307 resulting in an amino acid Ala being substituted for Thr at position 103. The E. coli F18-resistant pigs showed presence of the a nucleotide on both alleles (AA genotype), whereas pigs susceptible to E. coli F18 had either the heterozygous $A G$ genotype or the homozygous GG genotype $[7,8]$.

However, previous investigations conducted by Chinese scientists have shown that the polymorphism of FUT1 gene at nucleotide 307 only displays in foreign pig breeds and hybrid lines bred with foreign lineages such as the Sutai pig. There was no AA genotype or even the AG genotype in most Chinese domestic pig breeds, except the Lingao pig breed which carried a small proportion of AG genotype [9-11].

The Sutai pig is a new hybrid between the Duroc and Taihu breeds that produces high-quality lean meat. In previous studies, we identified a few FUT1 AG animals $(9.2 \%)$ in a Sutai pig population and selectively bred them to generate the prized Sutai FUT1 AA individuals (ETEC F18 resistant). After five years of continuous selection and breeding, the E. coli F18-resistant resource population with AA genotype was established [12]. Simultaneously, we also constructed a type V secretion system to express ETEC F18 adhesin. The display of functional adhesin through the type $V$ secretion system was combined with receptor binding experiments to further analyze and verify the resistance 
to the ETEC F18 strain among this E. coli F18-resistant resource population [13].

Following the initial studies on the FUT1 gene, examination on the expression of FUT1 gene in imported, hybrid and Chinese domestic breeds (Yorkshire, Sutai and Meishan) is an attractive route for the analysis of differences in genetic resistance and mechanism to E. coli F18. And it can be also be helpful in the development of research on the E. coli F18 receptor and the mechanism of resistance to E. coli F18 in Chinese native breeds.

\section{Material and Methods}

\section{Experimental materials and sample collection}

Yorkshire, Sutai and Meishan pig were collected from Engineering Research Centre for Molecular Breeding of Pig in Changzhou City, Jiangsu Province, E. coli F18-resistant population in Suzhou Taihu Pig Breeding Center and Meishan Pig Conservation Breeding Company, respectively. Each group included eight weaning piglets aged 35 days old from different litters, which were healthy and the growth characteristics are basically identical. After sacrifice, the following organs, heart, liver, spleen, lung, kidney, stomach, thymus, lymph node, jejunum, duodenum and muscle, were collected in $1.5 \mathrm{ml}$ Eppendorf nuclease-free tubes and stored immediately in liquid nitrogen and then placed in a low temperature freezer $\left(-80^{\circ} \mathrm{C}\right)$ until further study.

\section{Primer sequences}

Real-time PCR primers sequences of FUT1 gene were designed as P1: 5'- CAGATAAGCGAGGCCGTCATT-3' and P2: 5'-TTGCAGCCCACAAAAAGCA-3' using Primer Express 2.0 Software. For specificity, sequences of primers were aligned using the GenBank BLAST program, available online (www.ncbi.nlm. nih.gov). Each primer was designed to span the exon boundaries in order to avoid genomic DNA contamination and designed to produce amplification fragment of $100 \mathrm{bp}$ in length. In this study, GAPDH was used as a housekeeping gene to normalize the threshold cycle $(\mathrm{Ct})$ values of other tissue products with primer sequences as P1: 5' - ACATCATCCCTGCTTCTACTGG-3' and P2: 5' CTCGGACGCCTGCTTCAC -3 '.

\section{RNA extraction}

Total RNA was extracted from homogenized tissues (50-100 mg) using Trizol reagent (TaKaRa Biotechnology Dalian Co., Ltd). Precipitated RNA was resuspended in $20 \mu \mathrm{L}$ of RNase-free $\mathrm{H}_{2} \mathrm{O}$ and then stored at $-80^{\circ} \mathrm{C}$. RNA quality and quantity were assessed by $2.2 \mathrm{M}$ denatural agarose electrophoresis and UV spectrophotometry, respectively. The total RNA with A260/A280 value should be about 2.0.

\section{Real-time PCR}

Total RNA (500 ng) was reverse transcribed in a final reaction volume of $10 \mu \mathrm{L}$ using Primer Script RT reagent Kit (TaKaRa Biotechnology Dalian Co., Ltd) containing $5 \mu \mathrm{L} 5 \times$ Primer Script Buffer, $0.5 \mu \mathrm{L}$ Primer Script RT Enzyme Mix I, $0.5 \mu \mathrm{L}$ Oligo (dT), $0.5 \mu \mathrm{L}$ random 6-mers and RNase-free $\mathrm{H}_{2} \mathrm{O}$. The cDNA was synthesized at $37^{\circ} \mathrm{C}$ for 15 min followed by a termination step at $85^{\circ} \mathrm{C}$ for $5 \mathrm{~s}$ and then stored at $-20^{\circ} \mathrm{C}$.

Real-time PCR amplification was performed in $20 \mu \mathrm{L}$ reaction mixtures containing $1 \mu \mathrm{L}$ cDNA, $0.4 \mu \mathrm{L} 50 \times$ ROX Reference Dye

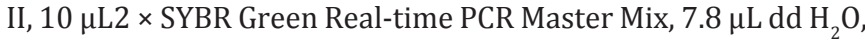
$0.4 \mu \mathrm{L}(10 \mu \mathrm{M})$ of each gene specific primers and GAPDH primers. PCR reactions were performed on the ABI 7500 Real-time PCR System. PCR cycling parameters were initially started at $95^{\circ} \mathrm{C}$ for $15 \mathrm{~s}$, and then $95^{\circ} \mathrm{C}$ for $5 \mathrm{~s}$ followed by $62^{\circ} \mathrm{C}$ for $30 \mathrm{~s}$ for 40 cycles. Dissociation curve analysis was performed at the end of 40 cycles to verify PCR product identity. Each sample was tested three times to obtain average data.

\section{Data processing and analysis}

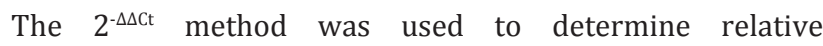
quantification [14] $(\Delta \mathrm{Ct}=$ the mean expression level of FUT1the mean expression level of $G A D P H$ ). The average expression level of FUT1 in the muscle tissues was defined as 1.0 so that the expression levels of this gene in other tissues could be quantified $(\Delta \Delta \mathrm{Ct}=\Delta \mathrm{Ct}$ of different tissue $-\Delta \mathrm{Ct}$ of muscle). Statistical analyses were carried out using SPSS 11.0 software. T-test was carried out to analyze the differentiation significance of mRNA expression in 11 tissues among three populations.

\section{Results}

\section{Results of real-time PCR}

The total RNA met the required standards for purity and was reverse-transcribed successfully. The cDNA was used for Real-time PCR. After PCR reaction, the ABI System automatically generated amplification curves related to changes of fluorescence and melting point curves. Melting point curves for FUT1 gene PCR primers have a single peak, which indicated that single PCR product was produced. Therefore, there was no indication of amplification of non-specific targets or primer dimers, which may complicate the quantification of target genes. The efficiency of the GAPDH PCR primers matched that of the FUT1 gene, which indicated that relative differences in target genes can be calculated according to the $2^{-\Delta \Delta C t}$ mathematical mode.

The results were shown in Table 1 and Figure 1 . The expression of FUT1 gene in 11 tissues in three different pig breeds shared almost the same characters. Relative to muscle (with a gene expression value of 1), FUT1 gene was found to be expressed in all the tissues in three breeds with relatively high expression in lung, stomach, liver, duodenum and jejunum. We observed that piglet lymph node, heart, thymus and muscle all expressed the FUT1 gene weakly.

On the whole, among Yorkshire, Meishan, and E. coli F18resistant Sutai groups, the expression of FUT1 gene was highest in Sutai pigs, followed by Yorkshire and Meishan pigs. There was a significant difference between the expression rates of Sutai and Meishan pigs together in stomach, duodenum and jejunum. Although most tissues of Yorkshire expressed FUT1 gene higher than Meishan pigs', there was no Significant difference between two groups. 
Table 1: Expression levels of FUT1 gene in 11 tissues in three different pig populations.

\begin{tabular}{|c|c|c|c|}
\hline Tissue & Meishan & Yorkshire & E. coli F18-resistant Sutai \\
\hline Heart & $2.018 \pm 0.910$ & $5.145 \pm 2.300$ & $5.260 \pm 2.264$ \\
\hline Liver & $34.615 \pm 13.719$ & $53.512 \pm 24.387$ & $90.040 \pm 49.205$ \\
\hline Spleen & $18.753 \pm 5.301$ & $41.890 \pm 6.405$ & $31.330 \pm 15.125$ \\
\hline Lung & $66.820 \pm 24.887$ & $87.830 \pm 38.216$ & $133.300 \pm 48.779$ \\
\hline Kidney & $18.102 \pm 2.666$ & $32.053 \pm 11.455$ & $25.620 \pm 10.670$ \\
\hline Stomach & $30.487 \pm 15.229^{a}$ & $96.052 \pm 35.522^{\mathrm{ab}}$ & $192.830 \pm 50.262^{b}$ \\
\hline Muscle & $1.000 \pm 0.000$ & $1.000 \pm 0.000$ & $1.000 \pm 0.000$ \\
\hline Thymus & $4.003 \pm 1.657$ & $4.436 \pm 2.302$ & $15.350 \pm 10.288$ \\
\hline Lymph node & $12.389 \pm 5.978$ & $15.564 \pm 4.082$ & $18.350 \pm 6.906$ \\
\hline Duodenum & $25.933 \pm 6.510^{a}$ & $57.693 \pm 31.369^{\mathrm{ab}}$ & $61.370 \pm 23.025^{b}$ \\
\hline Jejunum & $16.882 \pm 9.928^{a}$ & $20.404 \pm 6.999^{\mathrm{ab}}$ & $61.460 \pm 32.402^{b}$ \\
\hline
\end{tabular}

Note: Values in same organs with same letters indicate $P>0.05$, and those with different letters indicate $P<0.05$.

Value $=$ mean \pm SD .

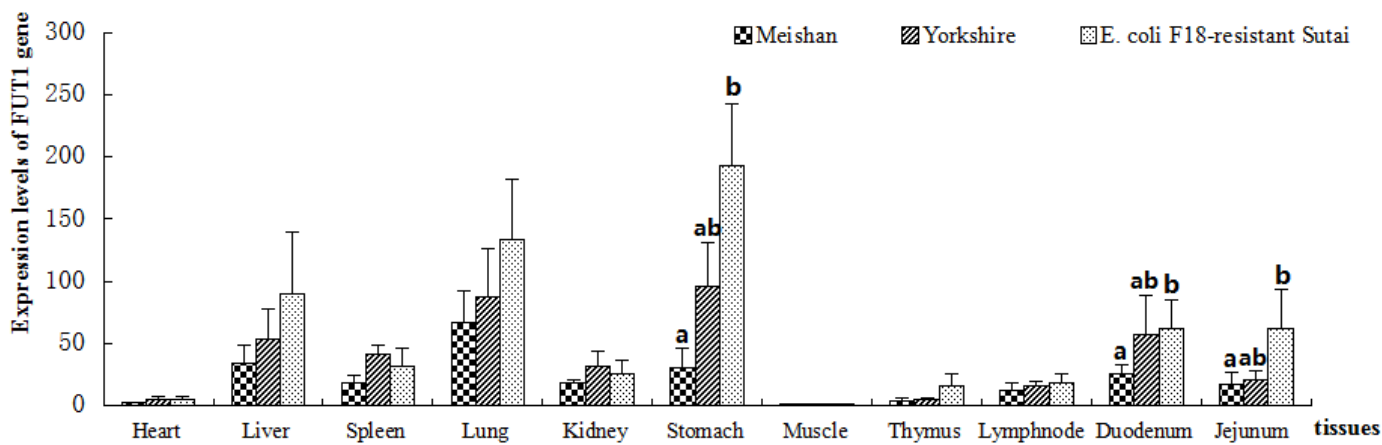

Figure 1: Expression levels of FUT1 gene in 11 tissues in three different pig populations.

Note: Values in same organs with same letters indicate $P>0.05$, and those with different letters indicate $P<0.05$.

Meishan pig, one of the Chinese native pig breeds, is well known all over the world for the high prolificacy and high adversity resistance. Compared the expression of FUT1 gene between Meishan and E. coli F18-resistant Sutai piglets, the result revealed that FUT1 gene expressed higher in the latter in all tissues. And the expression of FUT1 gene in stomach, duodenum and jejunum in E. coli F18-resistant Sutai piglets was significantly different from that in Meishan pigs.

\section{Discussion}

Newly weaned pigs suffer from post-weaning diarrhoea or oedema disease due to E. coli F18 infections [15]. The key point to the infection depends on the receptors expressed on the small intestinal epithelium. Recent findings demonstrate that the E.coli F18 receptor is known. The Fed F tip-adhesion of F18 fimbriae is bound to $\mathrm{ABH}$ type 1 glycosphingolipids, and the ABH type 1 $\mathrm{H}$ antigen is catalyzed by $\alpha 1$-fucosytransferase (FUT1) $[2,16,17]$. So the FUT1 gene has been identified as a candidate gene for controlling the expression of the enterotoxigenic E. coli F18 receptor. Being a good genetic marker, the M307 mutation in FUT1 gene was likely to control the resistance or susceptibility to E. coli F18 [7].
Here, we measured the expression of FUT1 gene in different pig breeds (Yorkshire, Sutai, and Meishan piglets). The Sutai pigs were established as E. coli F18-resistant. The results showed that there was a similar trend in tissue-specific expression of FUT1 gene. It expressed in all the tissues with relatively high expression in lungs, stomach, liver, duodenum and jejunum, which was in accordance with the result we've found before $[12,18])$. The previous researches in this field all proposed that FUT1 gene on chromosome 6 was closely linked to the E. coli F18 receptor [4,7]). Therefore, the relatively high expression in small intestine such as duodenum and jejunum implied its function in influencing the expression or the structure of E. coli F18receptor. In addition, the lower expression in piglet lymph node and thymus indicated that FUT1 gene might not participate in immune system directly.

What attracted our attention most was the different expression of FUT1 gene in 11 tissues among three pig breeds. Due to the fact that Meishan pig is Chinese native pig breed and only possess GG genotype at the position of M307 in FUT1 gene, all the individuals are sensitive to E. coli F18 in theory. Therefore, comparison of expression levels between the different breeds; Yorkshire, Meishan and Sutai. The Sutai pigs had the highest expression level of FUT1 gene in all tissues, followed by 
Yorkshire and Meishan pigs. The duodenum and jejunum, where the receptors of E. coli F18 are expressed, had higher expression levels of FUT1 in E. coli F18-resistant Sutai and Yorkshire breeds. It was possible that the expression level in three breeds was due to the innate genetic background and environment.

It has been clear that glycoproteins or glycolipids, expressed on the surface of the cells are the main receptors targeted by Gram-negative bacteria. The highly specific binding sites on these receptors are usually part of the sugar chains. The function of FUT1 gene implied more detailed relations between fucose transferred by FUT1 and the structure of E. coli F18 receptors. Bao [19]; Meijerink, et al. [6] reported that FUT1 enzyme was likely important in the synthesis of a structure which enables adhesion of $E$. coli F18 bacteria to small intestinal mucosa. Considering that this complement of proteins does not necessarily match to the complement of mRNA transcripts within the cells, we could speculate that the higher level of FUT1 gene was able to increase the proportions of the receptors' expression in F18-sensitive Sutai and Yorkshire, and result in their individual predisposition to disease. On the contrary, for Meishan pigs with relatively lower expression of FUT1 gene, there would be less chance of forms of receptors or being affected by toxins produced by E. coli F18.

In addition, it is well known that Meishan pigs have a character for higher stress resistance [20,21], such as the invasion of $E$. coli F18, even though they only harbour GG genotype, which was contradictory to the findings reported by Meijerink, et al [7]. Therefore, we made the comparison between Meishan and E. coli F18-resistant Sutai piglets. The expression of FUT1 gene in stomach, duodenum and jejunum in E. coli F18-resistant Sutai piglets was significantly higher than that in Meishan. We also know that the $307 \mathrm{G}>$ A mutation had no effect on the expression of FUT1 gene [12]. Those indicated that the function of FUT1 gene in influencing the E. coli F18 adhesion might be regulated by two ways, differential expression and amino acid change. This also suggested that there was a genetic difference in $E$. coli F18 resistance between Sutai and Meishan. Using genetic and enzymatic technology, Meijerink, et al. [7]; Meijerink [6] investigated that the levels of $\alpha(1,2)$-fucosyltransferase activity were significantly lower in intestinal mucosa of E. coli F18resistant pigs than in susceptible pigs. Moreover, lysates of CHO cells transfected with FUT1 constructs encoding threonine at amino acid position 103 also showed significantly reduced enzyme activity compared with constructs encoding alanine at this position. So it was possible that for foreign piglets, a favorable mutation occurred and reserved in the course of evolution in order to protect organism from the infection of E. coli F18 and meet with the hostile environment. Under natural condition, the frequency of the gene mutation in foreign pigs, such as Duroc and Landrace, was around 0.20 , which was quite low $[10,22]$.

Meanwhile, for Meishan pigs, it also possesses the strong immune system that decreases the risk of $E$. coli F18 infection. As Coddens, et al. [23] reported, colonization begins after adhesion of the bacteria with their fimbriae to the small intestine and stops when anti-fimbrial antibodies can be detected in the intestinal lumen of the infected pig. So we could presume that there was no need for Meishan pigs to have the mutation by virtue of its original higher resistance and lower expression of FUT1 gene. This may be the reason why there were no polymorphisms of FUT1 gene in Chinese domestic pigs, which suggested a complicated immuneregulating mechanism and genetic heterogeneity.

In this study, we conducted the expression of FUT1 gene in Yorkshire, Meishan and Sutai piglets, and Sutai piglets were established as E. coli F18-resistant. It prompted the speculation that the high expression of FUT1 in jejunum and duodenum was probably responsible for the structure of E. coli F18 receptors, and despite that the $307 \mathrm{G}>\mathrm{A}$ mutation in FUT1 gene had no correlation with higher expression of the gene itself, it may inhibit the FUT1 activity and exerted a negative impact on the formation of the receptors. Apart from the reason for different genetic background, the variation in promoter of FUT1 gene, the methylation or the regulation by adjacent genes in the same pathway may lead to the lowest expression of FUT1 gene in Meishan pigs. And further investigations involving determination of $E$. coli $\mathrm{F} 18$ receptors and the mechanism of resistance to $E$. coli F18 in Chinese native breeds must be of great importance.

\section{Acknowledgements}

This work was supported and funded by Natural Science Foundation of the Jiangsu Higher Education Institutions of China (14KJA230003), National Natural Science Funds (31472066), Science and Technology Supporting Project of Jiangsu Province (BE2014357, BE2012330, BE2013345) and the Priority Academic Program Development of Jiangsu Higher Education Institutions (PAPD).

\section{References}

1. Vogeli P, Bertschinger HU, Stamm M. Evidence for linkage of genes of the halothane (HAL) linkage group to the genes specifying receptors for F18 fimbriated Escherichia coli strains causing oedema disease and post weaning diarrhoea in Swiss Landrace pigs. Anim Gene. 1996;27:321-328.

2. Coddens A, Diswall M, Anqstrom J, Breimer ME, Goddeeris B, Cox $\mathrm{E}$, et al. Recognition of blood group $\mathrm{ABH}$ type 1 determinants by the FedF adhesin of F18-fimbriated Escherichia coli. J Biol Chem. 2009;284(15):9713-9726.

3. Sako F, Gasa S, Makita A, Hayashi A, Nozawa S. Human blood group glycosphingolipids of porcine erythocytes. Arch Biochem Biophys. 1990;278:228-237.

4. Beyer TA, Sadler JE, Hill RL. Purification to homogeneity of H blood group $\beta$-galactoside $\alpha 1$ leads to 2 Fucosyltransferase from porcine sub maxillary gland. J Biol Chem. 1980;255:5364-5372.

5. Thurin J, Blaszczyk-Thurin M. Porcine submaxillary gland GDP-Lfucose: Beta-D-galactoside alpha-2-L-fucosyltransferase is likely a counterpart of the human Secretor gene-encoded blood group transferase. J Biol Chem. 1995;270:26577-26580.

6. Meijerink E, Neuenschwander S, Fries R, Dinter A, Bertschinger HU, Stranzinger G, et al. A DNA polymorphism influencing alpha(1,2)fucosyltransferase activity of the pig FUT1 enzyme determines susceptibility of small intestinal epithelium to Escherichia coli F18. Immunogenetics. 2000;52(1-2):129-136.

7. Meijerink E, Fries R, Vögeli P, Masabanda J, Wigger G, Stricker C, et 
al. Two alpha(1,2) fucosyltransferase genes on porcine chromosome $6 q 11$ are closely linked to the blood group inhibitor (S) and Escherichia coli F18 receptor (ECF18R) loci. Mamm Genome. 1997;8:736-741.

8. Vogeli P, Meijerink E, Fries R, Neuenschwander S, Vorlander $\mathrm{N}$ Stranzinger G, et al. A molecular test for the detection of E. coli F18 receptors: a breakthrough in the struggle against oedema disease and post-weaning diarrhoea in swine. Schweiz Arch Tierheilkd. 1997;139:479-484

9. Shi QS, Xie XM, Liu XC, Huang SQ, He CQ. Experimental results on enterotoxigenic E. coli F18 receptor genotypes. Yi Chuan 2002;24(6):656-658

10. Yan XM, Ren J, Guo YM, Ding NS, Chen KF, Gao J, et al. Research on the genetic variations of 1,2-fucosytransferase (FUT1) gene in 26 pig breeds. Yi Chuan Xue Bao. 2003;30(9):830-834.

11. Bao WB, Wu SL, Musa HH, Zhu GQ Chen GH. Genetic variation at the alpha (1) fucosyltransferase (FUT1) gene in Asian wild boar and Chinese and Western commercial pig breeds. J Anim Breed Genet. 2008;125(6):427-430.

12. Bao WB, Ye L, Pan ZY, Zhu J, Du ZD, Zhu GQ, et al. The effect of mutation at M307 in FUT1 gene on susceptibility of Escherichia coli F18 and gene expression in Sutai piglets. Mol Biol Rep. 2012;497:336-339.

13. Wu SL, Yuan ZW, Ju HP, Huang XG, Hua JD, Shen JL, et al. Polymorphisms of the FUT1 gene M307 locus in post-weaning Sutai breed piglet and resistance to F18 fimbrial Escherichia coli in vitro. Chinese Journal of Preventive Veterinary Medicine. 2007;29:783-787.

14. Livak KJ, Schmittgen TD. Analysis of relative gene expression data using real time quantitative PCR and the 2 (-Delta Delta C(T)) Method. Methods. 2001;25:402-408.

15. Bertschinger HU, Bauchmann M, Mettler C, Pospischil A, Schraner EM, et al. Adhesive fimbriae produced in vivo by Escherichia coli 0139: K12 (B):H1 associated with enterotoxaemia in pigs. Vet Microbiol 1990;25:267-281.
16. Moonens K, Bouckaert J, Coddens A, Tran T, Panjikar S, De Kerpel M, et al. Structural insight in histo-blood group binding by the F18 fimbrial adhesin FedF Mol Microbiol. 2012;86(1):82-95. doi: 10.1111/j.13652958.2012.08174.x.

17. Lonardi E, Moonens K, Buts L, de Boer AR, Olsson JD, Weiss MS, et al. Structural Sampling of Glycan Interaction Profiles Reveals Mucosal Receptors for Fimbrial Adhesins of Enterotoxigenic Escherichia coli. Biology (Basel). 2013;2(3):894-917. doi: 10.3390/biology2030894.

18. Bao WB, Ye L, Zi C, Su XM, Pan ZY, Zhu J, et al. Study on the agedependent tissue expression of FUT1 gene in porcine and its relationship to E. coli F18 receptor. Gene. 2012a ;497:336-339. doi: 10.1016/j.gene.2012.01.035

19. Bao WB, Ye L, Pan ZY, Zhu J, Du ZD, , Zhu GQ, et al. Microarray analysis of differential gene expression in sensitive and resistant pig to Escherichia coli F18. Anim Genet. 2012b;43(5):525-534. doi: 10.1111/j.1365-2052.2011.02287.x.

20. Duchet-Suchaux MF, Bertin AM, Menanteau PS. Susceptibility of Chinese Meishan and European large white pigs to enterotoxigenic Escherichia coli strains bearing colonization factor K88, 987P, K99, or F41. Am J Vet Res. 1991;52(1):40-44.

21. Michaels RD, Whipp SC, Rothschild MF. Resistance of Chinese Meishan, Fengjing, and Minzhu pigs to the K88ac+ strain of Escherichia coli. Am J Vet Res. 1994;55(3):33-38.

22.Zhang YH, Zhou ZX, Cao GQ. FUT1 gene polymorphism and its association with litter size in pigs. Chinese Journal of Comparative Medicine. 2007;29(1):52-56.

23. Coddens A, Verdonck F, Tiels P, Rasschaert K, Goddeeris BM, Cox E, et al. The age-dependent expression of the E. coli F18 receptor on porcine gut epithelial cells is positively correlated with the presence of histo-blood group antigens. Vet Microbiol. 2007;122(3-4):332-341. 